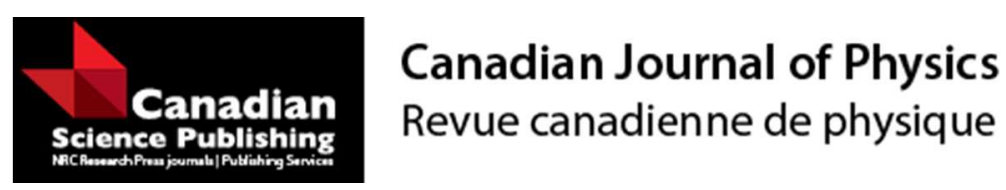

\title{
Renormalization Scheme Dependence and Renormalization Group Summation
}

\begin{tabular}{|r|l|}
\hline Journal: & Canadian Journal of Physics \\
\hline Manuscript ID & cjp-2016-0780.R4 \\
\hline Manuscript Type: & Article \\
\hline Date Submitted by the Author: & 23-Aug-2017 \\
\hline Complete List of Authors: & $\begin{array}{l}\text { Chishtie, F.A.; University of Western Ontario, Applied Mathematics } \\
\text { McKeon, D.G.C.; University of Western Ontario, }\end{array}$ \\
\hline $\begin{array}{r}\text { Keyword: } \\
\text { Is the invited manuscript for } \\
\text { consideration in a Special } \\
\text { Issue? : }\end{array}$ & N/A \\
\hline \multicolumn{2}{|l}{} \\
\hline
\end{tabular}




\title{
Renormalization Scheme Dependence and Renormalization Group Summation
}

\author{
F.A. Chishtie ${ }^{1}$ and D.G.C. McKeon ${ }^{2,3}$ \\ ${ }^{1}$ Department of Physics and Astronomy, The University of Western Ontario, \\ London, ON N6A 3K7, Canada \\ ${ }^{2}$ Department of Applied Mathematics, The University of Western Ontario, \\ London, ON N6A 5B7, Canada \\ ${ }^{3}$ Department of Mathematics and Computer Science, Algoma University, \\ Sault St.Marie, ON P6A 2G4, Canada
}

email: dgmckeo2@uwo.ca

Key Words: renormalization group, temperature, instantons, sum rules PACS No.: $12.38 \mathrm{Cy}$

\begin{abstract}
We consider logarithmic contributions to the free energy, instanton effective action and Laplace sum rules in QCD that are a consequence of radiative corrections. Upon summing these contributions by using the renormalization group, all dependence on the renormalization scale parameter $\mu$ cancels. The renormalization scheme dependence in these processes is examined, and a renormalization scheme is found in which the effect of higher order radiative corrections is absorbed by the behaviour of the running coupling.
\end{abstract}

\section{Introduction}

The process of renormalization in quantum field theory induces at finite order in perturbation theory a dependence on arbitrary parameters. The requirement that physical processes be independent of these parameters leads to the renormalization group (RG) equations [1-3]. Irrespective of the renormalization scheme (RS) used to excise divergences from a calculation, one of these arbitrary parameters is the renormalization mass scale parameter $\mu$. When one uses a mass-independent RS [4-5] there is also arbitrariness associated with the expansion coefficients of the RG functions associated with the couplings [6] and mass parameters [7] occurring in the theory. The RS dependence https://mc06.manuscriptcentral.com/cjp-pubs 
of the cross section for $e^{+} e^{-}$scattering $[8,9]$ as well as the semi-leptonic decay rate of the $b$ quark [10] has been considered.

In this paper we apply the approach used in refs. [8-10] to a number of other processes. In particular, we consider the free energy in thermal QCD, the effective action induced by instantons, and the Laplace sum rules for scalar gluon currents in QCD. The point of the calculations is to illustrate two things. The first is to show that if the RG equation associated with the arbitrary mass scale $\mu$ can be used to sum all logarithmic corrections to a processes that are due to radiative effects, then the explicit and implicit dependence on $\mu$ entirely cancels. The second is to show that the RS dependency of these RG summed results can be exploited to reduce the perturbative contributions to a finite number of terms in a series expansion in powers of the coupling; there is a particular mass independent RS in which all higher loop calculations serve only to determine the behaviour of the running parameters that characterize the theory.

\section{Thermal Free Energy}

The thermal free energy in QCD with $n_{f}$ quark flavours at temperature $T$ is given by [11-13],

$$
\mathcal{F}=\mathcal{F}_{0}\left[1+\sum_{n=0}^{\infty}\left(R_{n}(U) a^{n+1}+S_{n}(U) a^{n+3 / 2}+T_{n}(U) a^{n+2} \ln a\right)\right]
$$

with

$$
R_{n}(U)=\sum_{m=0}^{\infty} r_{n+m, m} U^{m}, \quad S_{n}(U)=\sum_{m=0}^{\infty} s_{n+m, m} U^{m}, \quad T_{n}(U)=\sum_{m=0}^{\infty} t_{n+m, m} U^{m}
$$

where $a=\alpha_{s} / \pi$ is the couplant, $\mathcal{F}_{0}$ is the ideal gas value and $U=a \ln \left(\frac{\mu}{2 \pi T}\right) \equiv a L$ (one could always absorb the factor of $\ln (2 \pi)$ appearing in $U$ into a redefinition of the coefficients $r_{m n}, s_{m n}$ and $\left.t_{m n}\right)$. At finite order, this result shows strong dependence on the renormalization scale $\mu$, both through the explicit dependence on $\ln \frac{\mu}{2 \pi T}$, and implicitly through $a(\mu)$ where

$$
\begin{aligned}
\mu \frac{d a}{d \mu} & =\beta(a) \\
& =-b a^{2}\left(1+c a+c_{2} a^{2}+\ldots\right) .
\end{aligned}
$$

$\mathcal{F}$ has been computed when the sums in eq. (1) extend just to 1 . The exact expression for $\mathcal{F}$ is independent of $\mu$ and so we have the RG equation

$$
\left(\mu \frac{\partial}{\partial \mu}+\beta(a) \frac{\partial}{\partial a}\right) \mathcal{F}=0 .
$$

In ref. [14] it was shown that eq. (3) allows one to compute $R_{0}, S_{0}, T_{0}$ (the "leading-log" - $L L$ ) result once $b$ is known; from this and a knowledge of $c$ then $R_{1}, S_{1}, T_{1}$ (the "next-to-leading-log" 
- NLL) are determined. Following this, then $R_{n}, S_{n}, T_{n}$ (the $N^{n} L L$ result) are fixed by a set of nested equations provided $c_{n}$ is known (with the computed values of $R_{n}(0), S_{n}(0), T_{n}(0)$ serving as boundary conditions). These RG summed results, being closer to the exact value of $\mathcal{F}$ than purely perturbative results, exhibit a diminished dependence on $\mu$.

We now reconsider $R G$ summation of $\mathcal{F}$, following the approach of refs. [8,9]. If we now rewrite eq. (1) in the form

$$
\mathcal{F}=\mathcal{F}_{0}\left(1+\sum_{n=0}^{\infty} \Omega_{n}(a) L^{n}\right)
$$

where

$$
\Omega_{n}(a)=\sum_{m=0}^{\infty}\left[r_{n+m, n} a^{n+m+1}+s_{n+m, n} a^{n+m+\frac{3}{2}}+t_{n+m, n} a^{n+m+2} \ln a\right]
$$

then together eqs. (3-4) result in

$$
\Omega_{n}(a)=-\frac{\beta}{n} \frac{d}{d a} \Omega_{n-1}(a)
$$

which by eq. (2) can be written as

$$
\Omega_{n}\left(a\left(\ln \frac{\mu}{\Lambda}\right)\right)=-\frac{1}{n} \frac{d}{d \ln \left(\frac{\mu}{\Lambda}\right)} \Omega_{n-1}\left(a\left(\ln \frac{\mu}{\Lambda}\right)\right)
$$

where $\Lambda$ is related to be boundary condition on eq. (2) [6],

$$
\ln \left(\frac{\mu}{\Lambda}\right)=\int_{0}^{a} \frac{d x}{\beta(x)}+\int_{0}^{\infty} \frac{d x}{b x^{2}(1+c x)} .
$$

and where $b$ and $c$ appear in eq. (2). Together, eqs. $(4,6)$ result in

$$
\begin{aligned}
\mathcal{F} / \mathcal{F}_{0} & =\left[1+\sum_{n=0}^{\infty} \frac{(-L)^{n}}{n !}\left(\frac{d}{d\left(\ln \frac{\mu}{\Lambda}\right)}\right)^{n} \Omega_{0}\left(a\left(\ln \frac{\mu}{\Lambda}\right)\right)\right] \\
& =\left[1+\Omega_{0}\left(a\left(\ln \frac{\mu}{\Lambda}-\ln \frac{\mu}{2 \pi T}\right)\right)\right] \\
& =\left[1+\Omega_{0}\left(a\left(\ln \frac{2 \pi T}{\Lambda}\right)\right)\right]
\end{aligned}
$$

In eq. (9), all dependence of $\mathcal{F}$ on $\mu$ (both implicit and explicit) has cancelled.

In Fig. 1, a graph of $\mathcal{F} / \mathcal{F}_{0}$ vs $T$ is shown with $\Lambda=300 \mathrm{MeV}$ for the RG summed and scaleindependent result of eq. (9), the $L L$ and $N L L$ RG summed result with $\mu=1 G e V$ and $\mu=4 G e V$ as well as the perturbative results of eq. (1) with $n=0,1$. This shows that the pertubative results along with the RG summed are fairly dependent on the RG scale $\mu$, whereas the scale independent eq. (9) (which is in the MS scheme) demonstrates certain regions of $\mathrm{T}$ where it is bounded by the RG summed results, while being different, especially for low values of $T$. 
Together eqs. $(1,4)$ show that eq. (9) is of the form

$$
\mathcal{F}=\mathcal{F}_{0}\left[1+\sum_{n=0}^{\infty}\left(A_{n} a^{n+1}+B_{n} a^{n+3 / 2}+C_{n} a^{n+2} \ln a\right)\right]
$$

where $A_{n}=R_{n}(0), B_{n}=S_{n}(0), C_{n}=T_{n}(0)$ and $a=a\left(\frac{2 \pi T}{\Lambda}\right)$.

The free energy $\mathcal{F}$ is also independent of the $R S$ used. In any mass-independent renormalization scheme, $b$ and $c$ in eq. (2) are $R S$ independent while the expansion parameters $c_{i}(i \geq 2)$ can be used to characterize the $R S$ used. If

$$
\frac{d a}{d c_{i}}=\sigma_{i}(a) \quad(i \geq 2)
$$

then from the requirement

$$
\left(\frac{\partial}{\partial \mu} \frac{\partial}{\partial c_{i}}-\frac{\partial}{\partial c_{i}} \frac{\partial}{\partial \mu}\right) a=0
$$

it follows that $[6]$

$$
\begin{aligned}
\sigma_{i}(a) & =-b \beta(a) \int_{0}^{a} d x \frac{x^{i+2}}{\beta^{2}(x)} \\
& \approx a^{i+1}\left[\frac{1}{i-1}-c\left(\frac{i-2}{i(i-1)}\right) a+\frac{1}{i+1}\left(c^{2} \frac{i-2}{i}-c_{2} \frac{i-3}{i-1}\right) a^{2}+\ldots\right] .
\end{aligned}
$$

We now can use eqs. $(10,13)$ to see how $A_{n}, B_{n} C_{n}$ all depend on $c_{i}$. We see that if

$$
\begin{aligned}
& \frac{d \mathcal{F}}{d c_{i}}=0=\mathcal{F}_{0} \sum_{n=0}^{\infty}\left\{\frac{\partial A_{n}}{\partial c_{i}} a^{n+1}+\frac{\partial B_{n}}{\partial c_{i}} a^{n+\frac{3}{2}}+\frac{\partial C_{n}}{\partial c_{i}} a^{n+2} \ln a\right. \\
&+\sigma_{i}(a)\left[(n+1) A_{n} a^{n}+\left(n+\frac{3}{2}\right) B_{n} a^{n+\frac{1}{2}}\right. \\
&\left.\left.+((n+2) \ln a+1) a^{n+1} C_{n}\right]\right\}
\end{aligned}
$$

is satisfied at each order in $a^{m} \ln ^{n} a$, it follows that

$$
\begin{gathered}
\frac{\partial A_{0}}{\partial c_{i}}=\frac{\partial B_{0}}{\partial c_{i}}=\frac{\partial C_{0}}{\partial c_{i}}=0 \\
\frac{\partial A_{1}}{\partial c_{i}}=\frac{\partial B_{1}}{\partial c_{i}}=\frac{\partial C_{1}}{\partial c_{i}}=0 \\
\frac{\partial A_{2}}{\partial c_{i}}+A_{0} \delta_{2}^{i}=\frac{\partial B_{2}}{\partial c_{i}}+\frac{3}{2} B_{0} \delta_{2}^{i}=\frac{\partial C_{2}}{\partial c_{i}}+2 C_{0} \delta_{2}^{i}=0 \\
\frac{\partial A_{3}}{\partial c_{i}}+\left(2 A_{1}+C_{0}\right) \delta_{2}^{i}+\frac{1}{2} A_{0} \delta_{3}^{i}=\frac{\partial B_{3}}{\partial c_{i}}+\frac{5}{2} B_{1} \delta_{2}^{i}+\frac{3}{4} B_{0} \delta_{3}^{i} \\
=\frac{\partial C_{3}}{\partial c_{i}}+3 C_{1} \delta_{2}^{i}+C_{0} \delta_{3}^{i}=0
\end{gathered}
$$


etc.

Eqs. (16-19) can be integrated to give

$$
\begin{gathered}
A_{0,1}=\alpha_{0,1} \quad B_{0,1}=\beta_{0,1} \quad C_{0,1}=\gamma_{0,1} \\
A_{2}=\alpha_{2}-\alpha_{0} c_{2} \quad B_{2}=\beta_{2}-\frac{3}{2} \beta_{0} c_{2} \quad C_{2}=\gamma_{2}-2 \gamma_{0} c_{2} \\
A_{3}=\alpha_{3}-\left(2 \alpha_{1}+\gamma_{0}\right) c_{2}-\frac{1}{2} \alpha_{0} c_{3} \quad B_{3}=\beta_{3}-\frac{5}{2} \beta_{1} c_{2}-\frac{3}{4} \beta_{0} c_{3} \quad C_{3}=\gamma_{3}-3 \gamma_{1} c_{2}-\gamma_{0} c_{3}
\end{gathered}
$$

etc.

In eqs. (20-22), $\alpha_{k}, \beta_{k}$ and $\gamma_{k}$ are constants of integration and consequently are $R S$ invariants. Once $A_{k}, B_{k}, C_{k}$ and $\beta(a)$ have been explicitly calculated in a particular $R S$ to order $N$ in the loop expansions, then $\alpha_{k}, \beta_{k}, \gamma_{k}$ can be found from eqs. (20-22) to order $N-1$. Two particular schemes then suggest themselves. One is the "'t Hooft scheme" [15] in which $c_{k}=0(k \geq 2)$ in which case $A_{k}=\alpha_{k}, B_{k}=\beta_{k}$ and $C_{k}=\gamma_{k}$. A second scheme is one in which either $A_{k}, B_{k}$ or $C_{k}$ vanish for $k \geq 2$. If $A_{k}=0(k \geq 2)$ then by eqs. $(21,22)$ the $\beta$ function expansion coefficients in eq. (2) are fixed,

$$
c_{2}=\frac{\alpha_{2}}{\alpha_{0}} \quad c_{3}=\frac{2}{\alpha_{0}}\left[\alpha_{3}-\left(2 \alpha_{1}+\gamma_{0}\right)\left(\frac{\alpha_{2}}{\alpha_{0}}\right)\right]
$$

etc.

and so

$$
A_{2}=0, \quad B_{2}=\beta_{2}-\frac{3}{2} \frac{\beta_{0} \alpha_{2}}{\alpha_{0}}, \quad C_{2}=\gamma_{2}-2 \frac{\gamma_{0} \alpha_{2}}{\alpha_{0}}
$$

etc. A similar approach can be used when considering a $R S$ in which $B_{k}=0(k \geq 2)$ or $C_{k}=0(k \geq$ $2)$.

\section{Instanton Effective Action}

The form of the effective action in an $S U(2)$ gauge theory with $n_{f}$ flavours is

$$
\mathcal{L}_{\text {eff }} \sim K \int_{0}^{\infty} d \rho \rho^{-5+3 n_{f}} \exp \left\{-\frac{8 \pi^{2}}{g^{2}} S\right\}\left(a \equiv \frac{g^{2}}{4 \pi^{2}}\right) .
$$

In eq. (25), the scale parameter $\rho$ is the instanton size and $S$ has been computed to one loop order [16]. In general, $S$ has the form

$$
S=\sum_{n=0}^{\infty} \sum_{m=0}^{n} T_{n, m} a^{n} \ln ^{m}(\mu \rho) \quad\left(T_{0,0}=1\right) .
$$

Since $\mathcal{L}_{\text {eff }}$ satisfies the $R G$ equation

$$
\left(\mu \frac{\partial}{\partial \mu}+\beta(a) \frac{\partial}{\partial a}\right) \mathcal{L}_{e f f}=0
$$


the functions $S_{n}(a L) \quad(L \equiv \ln (\mu \rho))$ can be calculated iteratively where

$$
S_{n}(a L)=\sum_{m=0}^{\infty} T_{n+m, m}(a L)^{m} \quad\left(S_{n}(0)=T_{n, 0}\right) .
$$

This has been considered in ref. [17]. However, if only a finite number of terms are kept in the expansion of eq. (26), $S$ will retain at least a residual dependence on $\mu$.

This dependence can be shown to cancel if one were to write (in much the same way as eq. (4)),

$$
S=\sum_{n=0}^{\infty} A_{n}(a) L^{n}
$$

where now

$$
A_{n}(a)=\sum_{m=0}^{\infty} T_{n+m, n} a^{n+m} \quad(n=0,1,2, \ldots) .
$$

Then as by eq. (27)

$$
\left(\mu \frac{\partial}{\partial \mu}+\beta(a) \frac{\partial}{\partial a}\right)\left(\frac{1}{a} \sum_{n=0}^{\infty} A_{n}(a) L^{n}\right)=0
$$

it follows that

$$
\frac{1}{a} A_{n}(a)=\frac{-1}{n} \beta(a) \frac{d}{d a}\left(\frac{1}{a} A_{n-1}(a)\right)
$$

and so, just as eq. (9) follows from eq. (5), we see that eq. (32) results in

$$
\mathcal{L}_{e f f}=K \int_{0}^{\infty} d p \rho^{-5+3 n_{f}} \exp \left\{\frac{-2}{a\left(\ln \frac{1}{\rho \Lambda}\right)} A_{0}\left(a\left(\ln \frac{1}{\rho \Lambda}\right)\right)\right\} .
$$

As with eq. (9), all the implicit and explicit dependence of $\mathcal{L}_{\text {eff }}$ on $\mu$ has cancelled upon doing the $R G$ sum.

We now see that by eq. (26)

$$
\frac{1}{a} A_{0}(a)=\frac{1}{a}\left(1+\sum_{n=1}^{\infty} T_{n} a^{n}\right)
$$

where $a=a\left(\ln \frac{1}{\rho \Lambda}\right)$ and $T_{n} \equiv T_{n, 0}$. As $\mathcal{L}_{\text {eff }}$ is $R S$ independent, then as with eq. (15)

$$
\frac{d}{d c_{i}}\left(\frac{1}{a} A_{0}(a)\right)=0=\sum_{n=1}^{\infty}\left(\frac{\partial T_{n}}{\partial c_{i}} a^{n-1}\right)+\sigma_{i}(a)\left(\frac{-1}{a^{2}}+\sum_{n=1}^{\infty}(n-1) T_{n} a^{n-2}\right)
$$

which is satisfied order by order in $a$ provided

$$
\frac{\partial T_{1}}{\partial c_{i}}=0
$$




$$
\begin{gathered}
\frac{\partial T_{2}}{\partial c_{i}}-\delta_{2}^{i}=0 \\
\frac{\partial T_{3}}{\partial c_{i}}-\frac{1}{2} \delta_{3}^{i}=0 \\
\frac{\partial T_{4}}{\partial c_{i}}-\frac{1}{3} \delta_{4}^{i}+\frac{c}{6} \delta_{3}^{i}+\left(T_{2}-\frac{c_{2}}{3}\right) \delta_{2}^{i}=0
\end{gathered}
$$

etc.

Solving for $T_{i}$ in turn from eq. (36) leads to

$$
\begin{gathered}
T_{1}=\tau_{1} \\
T_{2}=\tau_{2}+c_{2} \\
T_{3}=\tau_{3}+\frac{c_{3}}{2} \\
T_{4}=\tau_{4}+\frac{c_{4}}{3}-\frac{c c_{3}}{6}-\frac{c_{2}^{2}}{3}-\tau_{2} c_{2}
\end{gathered}
$$

etc.

In eq. (37), the $\tau_{i}$ are constants of integration and hence are $R S$ invariants. The 't Hooft $R S$ in which we choose $c_{i}=0(i \geq 2)$ leads to

$$
T_{i}=\tau_{i} \quad(i \geq 1)
$$

In a second $R S, T_{i}=0 \quad(i \geq 2)$ so that

$$
\begin{gathered}
c_{2}=-\tau_{2} \\
c_{3}=-2 \tau_{3} \\
c_{4}=-3\left(\tau_{4}+\frac{c \tau_{3}}{3}+\frac{2 \tau_{2}^{2}}{3}\right)
\end{gathered}
$$

etc.

with

$$
S=1+\tau_{1} a\left(\ln \frac{1}{\rho \Lambda}\right)
$$

there being no higher order contributions to $S$ in this scheme.

Substitution of eq. (40) into eq. (25) leads to

$$
\mathcal{L}_{e f f} \sim K \int_{0}^{\infty} d \rho \rho^{-5+3 n_{f}} \exp -2\left[\frac{1}{a\left(\ln \frac{1}{\rho \Lambda}\right)}+\tau_{1}\right]
$$

As $\rho \rightarrow 0^{+}$, at one loop order by eqs. $(2,7)$

$$
a\left(\ln \frac{1}{\rho \Lambda}\right) \longrightarrow \frac{1}{b \ln \left(\frac{1}{\rho \Lambda}\right)}
$$


on account of asymptotic freedom, and thus at the lower end of integration in eq. (41)

$$
\rho^{-5+3 n_{f}} \exp \left(\frac{-2}{a\left(\ln \frac{1}{\rho \Lambda}\right)}\right) \longrightarrow \rho^{-5+3 n_{f}}(\rho \Lambda)^{2 b}
$$

As for $S U(2), b=\left(\frac{22-2 n_{f}}{6}\right)$ we see that the integral in eq. (41) is convergent at the lower limit of integration for all values of $n_{f}$ in this $R S$. As $\rho \rightarrow \infty$, we anticipate that $1 / a \rightarrow 0$ and so for $n_{f}$ sufficiently small we see that at the upper limit of integration the integral in eq. (41) also converges, though if $a$ has an infrared fixed point, a divergence at the upper limit of integration would occur [27]. Having the integral in eq. (41) converge at both limits of integration is not what one expects when considering one-loop contributions to $S$ [16] or the leading-log contributions to $S$ [17].

\section{QCD Laplace Sum Rules for Scalar Gluon Currents}

With the scalar gluon operator

$$
j_{G}(x)=-\frac{2 \pi \beta(a)}{a b} G_{\mu \nu}^{a}(x) G^{a \mu \nu}(x)
$$

the correlation function

$$
\Pi_{G}\left(p^{2}\right)=i \int d y e^{i p \cdot y}<0\left|T j_{G}(y) j_{G}(0)\right| 0>
$$

is used to define the Laplace sum rule

$$
\mathcal{L}_{k}^{\text {pert }}(\tau)=\frac{1}{\pi} \int_{0}^{\infty} d s s^{k+2} e^{s \tau} I_{M} \Pi_{G}^{\text {pert }}(s) .
$$

It is now possible to make the expansion $[18,19]$

$$
\mathcal{L}_{k}^{\text {pert }}(\tau)=\frac{a_{k} a^{2}}{\tau^{k+3}}\left[1+\sum_{n=1}^{\infty} \sum_{m=0}^{n} T_{n, m}^{(k)} a^{n} L^{m}\right] \quad(L=\ln (\sqrt{\tau} \mu)) .
$$

As with $\mathcal{F}$ in eq. (1) and $\mathcal{L}_{\text {eff }}(25), \mathcal{L}_{k}^{\text {pert }}$ in eq. (46) has explicit and implicit dependence on $\mu$ at any finite order of perturbation theory that cancels upon summing all perturbative effects. In ref. [19] it is shown how summation of $N^{p} L L$ contributions to $\mathcal{L}_{k}^{\text {pert }}$ (using the computed values of $b, c, \ldots c_{p}$ in eq. (2) and $T_{p, 0}^{(k)}$ in eq. (47)) considerably reduces the $\mu$ dependence of $\mathcal{L}_{k}^{\text {pert }}$ from that of the purely perturbative result to order $(p+1)$ in the loop expansion. It is now possible to show how all of the $\mu$ dependence of $\mathcal{L}_{k}^{\text {pert }}$ can be made to cancel upon summing all logarithmic effects. To do this, we follow eqs. $(4,29)$ and define

$$
A_{n}^{(k)}(a)=\sum_{m=0}^{\infty} T_{n+m, n}^{(k)} a^{n+m+2} \quad(n=0,1,2, \ldots)
$$


where $T_{0,0}=1$ so that eq. (47) becomes

$$
\mathcal{L}_{k}^{p e r t}(\tau)=\frac{a_{k}}{\tau^{k+3}} \sum_{n=0}^{\infty} A_{n}^{(k)}(a) L^{n}
$$

The $R G$ equation

$$
\left(\mu \frac{\partial}{\partial \mu}+\beta(a) \frac{\partial}{\partial a}\right) \mathcal{L}_{k}^{p e r t}(\tau)=0
$$

leads to (as with eq. (6))

$$
A_{n}^{(k)}\left(a\left(\ln \frac{\mu}{\Lambda}\right)\right)=\frac{-\beta(a)}{n} \frac{d}{a\left(\ln \frac{\mu}{\Lambda}\right)} A_{n-1}^{(k)}\left(a\left(\ln \frac{\mu}{\Lambda}\right)\right)
$$

and so the sum in eq. (49) results in

$$
\mathcal{L}_{k}^{\text {pert }}(\tau)=\frac{a_{k}}{\tau^{k+3}} A_{0}^{(k)}\left(a\left(\ln \frac{1}{\sqrt{\tau} \Lambda}\right)\right)
$$

much like eqs. (9,33) above. By eq. (48), eq. (52) becomes

$$
\mathcal{L}_{k}^{\text {pert }}(\tau)=\frac{a_{k}}{\tau^{k+3}} a^{2}\left(1+\sum_{n=1}^{\infty} T_{n}^{(k)} a^{n}\right)
$$

where $a=a\left(\ln \frac{1}{\sqrt{\tau} \Lambda}\right)$ and $T_{n, 0}^{(k)} \equiv T_{n}^{(k)}$. In eq. (53) there is no dependence on $\mu$.

As in the preceding sections, the $R S$ dependence of the expansion coefficients $T_{n}^{(k)}$ in eq. (53) can be found by using the equation

$$
\left(\frac{\partial}{\partial c_{i}}+\sigma_{i}(a) \frac{\partial}{\partial a}\right) \mathcal{L}_{k}^{p e r t}=0
$$

With $\sigma_{i}(a)$ given by eq. (14), eq. (54) is satisfied order-by-order in a provided

$$
\begin{gathered}
\frac{\partial T_{1}^{(k)}}{\partial c_{i}}=0 \Rightarrow T_{1}^{(k)}=\lambda_{1}^{(k)} \\
\frac{\partial T_{2}^{(k)}}{\partial c_{i}}+2 \delta_{2}^{i}=0 \Rightarrow T_{2}^{(k)}=\lambda_{2}^{(k)}-2 c_{2} \\
\frac{\partial T_{3}^{(k)}}{\partial c_{i}}+3 T_{1}^{(k)} \delta_{2}^{i}+\delta_{3}^{i}=0 \Rightarrow T_{3}^{(k)}=\lambda_{3}^{(k)}-3 \lambda_{1}^{(k)} c_{2}-c_{3} \\
\frac{\partial T_{4}^{(k)}}{\partial c_{i}}+\left(4 T_{2}^{(k)}+\frac{2}{3} c_{2}\right) \delta_{2}^{i}+\left(\frac{3}{2} T_{1}^{(k)}-\frac{c}{3}\right) \delta_{3}^{i}+\frac{2}{3} \delta_{4}^{i}=0 \\
\Rightarrow T_{4}=\lambda_{4}^{(k)}-4 \lambda_{2}^{(k)} c_{2}+\frac{11}{3} c_{2}^{2}-\frac{3}{2} \lambda_{1}^{(k)} c_{3}+\frac{c c_{3}}{3}-\frac{2}{3} c_{4}
\end{gathered}
$$


etc.

where $\lambda_{i}^{(k)}$ is a constant of integration and consequently is a $R S$ invariant that can be determined once $T_{i}^{(k)}$ and $c_{i}$ have been evaluated in some mass independent $R S$ such as $M S$.

Again, in the 't Hooft $R S, c_{i}=0(i \geq 2)$ and so $T_{n}^{(k)}=\lambda_{n}^{(k)}$. In another $R S$, one can have $T_{i}=0(i \geq 2)$ so that by eq. (55)

$$
\begin{gathered}
c_{2}=\frac{1}{2} \lambda_{2}^{(k)} \\
c_{3}=\lambda_{3}^{(k)}-3 \lambda_{1}^{(k)} c_{2} \\
c_{4}=\frac{3}{2}\left(\lambda_{4}^{(k)}-4 \lambda_{2}^{(k)} c_{2}+\frac{11}{3} c_{2}^{2}-\frac{3}{2} \lambda_{1}^{(k)} c_{3}+\frac{c c_{3}}{3}\right)
\end{gathered}
$$

etc.

and $\mathcal{L}_{k}^{\text {pert }}(\tau)$ reduces to just two terms

$$
\mathcal{L}_{k}^{p e r t}=\frac{a_{k}}{\tau^{k+3}} a^{2}\left(1+\lambda_{1}^{(k)} a\right)
$$

Using the approach taken in [18], we estimate the scalar glueball mass bound $\frac{\mathcal{L}_{1}}{\mathcal{L}_{0}}$ in the MS scheme for the 4-loop perturbative calculation with $\xi=\mu \sqrt{\tau}=0.8$ and 1.2 , along with the $N^{3} L L$ RG summation and the scale independent result provided in eq. (53). The results are shown in Fig.2, where we find that remarkably the scale independent scalar glueball mass bound in the MS scheme is bounded within the $\mu$ dependent perturbative and RG summed results. In Figure 3, we depict the scale independent results for the mass bound for the MS, scheme 1 and scheme 2 as a function of $\tau$, where we find that the latter two schemes quite close to each other and all three schemes meeting at $\tau=1 \mathrm{GeV}^{-2}$ which corresponds to a scalar glueball mass bound of $\frac{\mathcal{L}_{1}}{\mathcal{L}_{0}} \leq 1.2$ $\mathrm{GeV}$.

\section{Discussion}

We have shown, by following the approach used in refs. [8-10], that if all logarithmic contributions to radiative corrections to QCD processes can be summed, then the explicit and implicit dependence on the renormalization scale parameter $\mu$ cancels. Furthermore, the $R S$ dependence can be analyzed and a particular $R S$ can be chosen so that either the $R G$ functions receive no contributions beyond two loop order, or the perturbative series in powers of the coupling terminates after a fixed number of terms.

Summing these logarithms is only possible if there is a sufficiently simple ansatz for the physical process being considered; it must be a power series in both the couplant and powers of the logarithm of $\mu$, with the power of the logarithm not exceeding that of the couplant (as in eqs. $(1,28,47)$ ). This seems to require that we use a mass independent $R S$ such as $M S$. An extension of the techniques that we have used so as to accommodate other renormalization schemes, especially when massive 
fields are being considered, is currently being examined. This would make it possible to consider electroweak processes, such as the semi-leptonic decay of the $b$ quark [10].

It would be most interesting to devise a way of summing the contribution of higher order effects in order to eliminate the explicit and implicit dependence on physical quantities on the RS dependent coefficients $c_{i}$. We are only able to arrange for the cancellation between the implicit and explicit dependence on $\mu$ because we know that the implicit dependence on $\mu$ takes a form such as appears in eqs. $(1,28,47)$ on account of the way in which the appropriate Feynman diagrams contribute. There is apparently no analogous way of handling any of the $c_{i}$.

We note that there are other approaches that have appeared in the literature in which the problem of reducing the $\mu$ dependence of results obtained using perturbative QCD has been addressed. In particular, the "principle of minimum sensitivity" (PMS) invokes the principle (of which there is no proof) that the "optimal" value of unphysical parameters are those that minimize changes in the quantity being computed at some fixed order in perturbation theory when these parameters are varied. The original version of PMS [6] has been refined [20, 21] to accommodate higher order perturbative calculations. It would be of interest to see if the optimal values of $\mu$ and $c_{i}$ obtained by applying PMS at finite fixed order of perturbation theory lead to results compatible with the approach used in this paper in which an all-order summation has been used. One might also consider application of the PMS procedure to optimize the value of the $c_{i}$ after one has computed the RG summed result.

In a second approach, which uses the "principle of maximum conformality" (PMC), dependence on the parameters $c_{i}(i \geq 2)$ is absorbed into the mass scale parameter $\mu$ at each order of perturbation theory [22-23]. Results obtained using PMS and PMC have been compared for various processes in ref. [21].

The question of the infrared (IR) limit of results obtained using perturbative QCD can also be addressed when using the renormalization group summation employed in this paper. One generally is concerned with the IR behaviour of $a(\mu)$ with $a(\mu)$ governed by eq. (2). However, it is apparent that at least in perturbation theory the IR limit of $a(\mu)$ is contingent upon the RS used; the values of $c_{i}$ affect this limit if $b<0$, at least when considering only a finite number of terms in the series expansion for $\beta(a)$. A discussion of this IR behaviour in the scheme in which $c_{i}=0$ (ie, $a(\mu)$ is the 't Hooft coupling [15]) appears in ref. [24, 25]; the IR behaviour when the four loop contributions to $\beta(a)$ in the $\overline{M S}$ scheme is used appears in ref. [26].

It should be kept in mind that when examining the IR behaviour of a physical quantity computed using perturbation theory (such as $\mathcal{F}$ in eq. (1)), one should consider not just how $a(\mu)$ behaves in this limit, but also the convergence behaviour of the infinite series in powers of $a(\mu)$ that arises in perturbation theory. Normally, the contribution of "renormalons" [15] is felt to result in such infinite series being at best asymptotic although renormalons are removed when using PMC [22,23]. In ref. [27] it is proposed that, in order to circumvent the need to consider the convergence behaviour of 
infinite series, it is appropriate to simply examine the IR behaviour of such finite series as appear in eqs. (41) and (57). In these cases the RG function $\beta(a)$ has the expansion coefficient $c_{i}$ fixed in terms of RS invariants ( $\tau_{i}$ in the case of eq. (41) and $\lambda_{i}^{(k)}$ in the case of eq. (57)) and so this IR behaviour will depend on the process being considered. In ref. [27] this approach to examining the IR behaviour of the cross section for $e^{+} e^{-} \rightarrow$ (hadrons) results in a well defined IR limit for the cross section when there are $n_{F}=3$ active flavours, even though the coupling $a$ diverges in the IR limit when it is computed using $\bar{M} S$ to four loop order.

\section{Acknowledgements}

The suggestions of T.N. Sherry were most helpful, as was a comment by Roger Macleod.

\section{References}

[1] E.C.G. Stueckelberg and A. Peterman, Helv. Phys. Acta 26, 499 (1953).

[2] M. Gell-Mann and F.E. Low, Phys. Rev. 95, 1300 (1954).

[3] N.N. Bogoliubov and D.V. Shirkov, Nuovo Cimento 3, 845 (1956).

[4] G. 't Hooft, Nucl. Phys. B61, 455 (1973).

[5] S. Weinberg, Phys. Rev. D8, 3497 (1973).

[6] P.M. Stevenson, Phys. Rev. D23, 2916 (1981).

[7] D.G.C. McKeon, Can. J. Phys. 61, 564 (1983); 59, 1327 (1981).

[8] D.G.C. McKeon, Phys. Rev. D92, 045031 (2015).

[9] F.A. Chishtie, D.G.C. McKeon and T.N. Sherry, Phys. Rev.D94, 054031 (2016).

[10] F.A. Chishtie, D.G.C. McKeon and T.N. Sherry, arXiv:1708.04219.

[11] P. Arnold and C.X. Zhai, Phys. Rev. D51, 1906 (1995).

[12] C.X. Zhai and B. Kastening, Phys. Rev. D52, 7232 (1995).

[13] E. Braaten and A. Nieto, Phys. Rev. D53, 3421 (1996).

[14] D.G.C. McKeon and A. Rebhan, Phys. Rev. D67, 027701 (2003). 
[15] G. 't Hooft, "The Whys of Subnuclear Physics" edited by A. Zichichi (Plenum, New York, 1979).

[16] G. 't Hooft, Phys. Rev. D14, 3432 (1976).

[17] M.R. Ahmady, V. Elias, D.G.C. McKeon, A. Squires and T.G. Steele, Nucl. Phys. B655, 221 (2003).

[18] F.A. Chishtie, V. Elias and T.G. Steele, Phys. Rev. D59, 105013 (1999).

[19] F.A. Chishtie, T.G. Steele and D.G.C. McKeon, Phys. Lett. B754, 43 (2016).

[20] P.M. Stevenson, Nucl. Phys. B868, 38 (2013).

[21] Y. Ma, X.G. Wu, H.H. Ma and H.Y. Han, Phys. Rev. D91, 034006 (2015).

[22] S.J. Brodsky, M. Mojaza and X.G. Wu, Phys. Rev. Lett. 110, 192001 (2013); ibid Phys. Rev. D89, 014027 (2014).

[23] S.J. Brodsky, H.B. Fu, H.H. Ma, Y. Ma, M. Mojaza, S.Q. Wang and X.G. Wu, Rep. Prog. Phys. 78, 126201 (2015).

[24] T. Banks and A. Zaks, Nucl. Phys. B196, 189 (1982).

[25] N.N. Khuri and O.A. McBryan, Phys. Rev. D20, 881 (1979).

[26] C. Pica and F. Sannino, Phys. Rev. D83, 035013 (2011).

[27] F.A. Chishtie and D.G.C. McKeon, Phys. Rev. D95, 116013 (2017). 


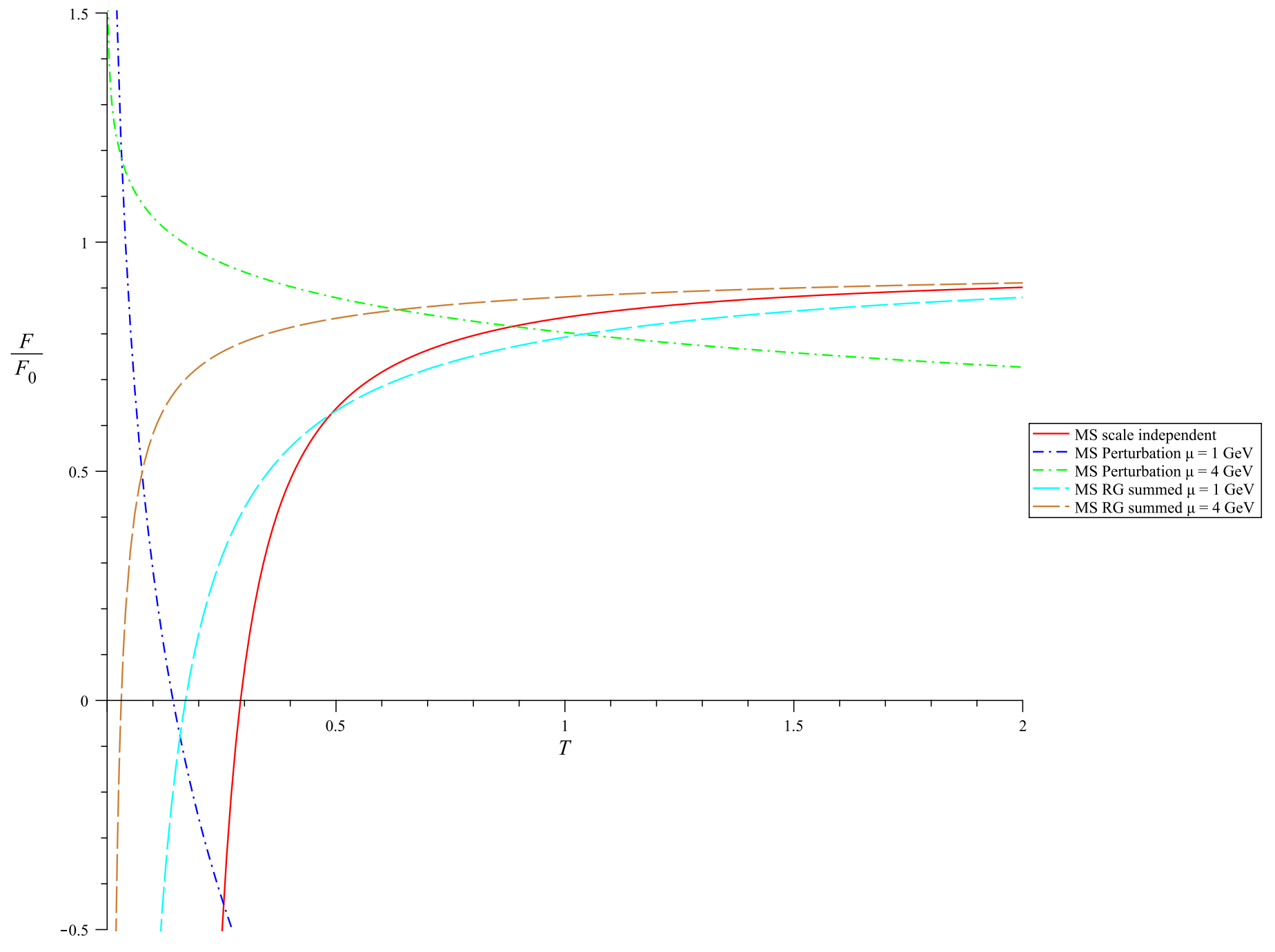

Figure 1: $\mathcal{F} / \mathcal{F}_{0}$ as a function of temperature $T$ with perturbative and $\mathrm{RG}$ summed results 


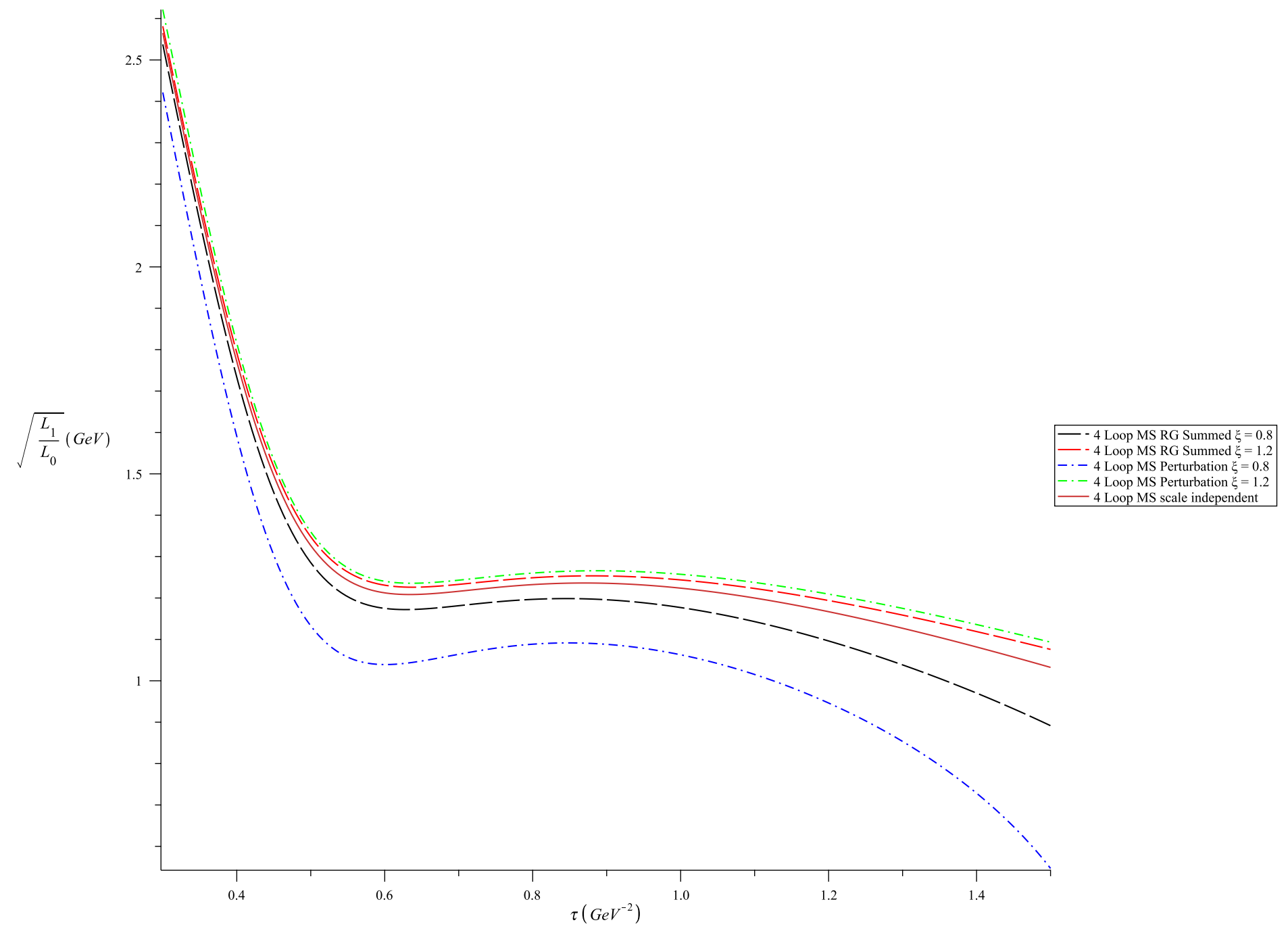

Figure 2: The $\mu$ dependence of the scalar glueball mass bound in MS scheme with truncated, RG summed and scale independent forms with respect to $\tau\left(G e V^{-2}\right)$ using values of $\xi=0.8$ and 1.2 respectively 


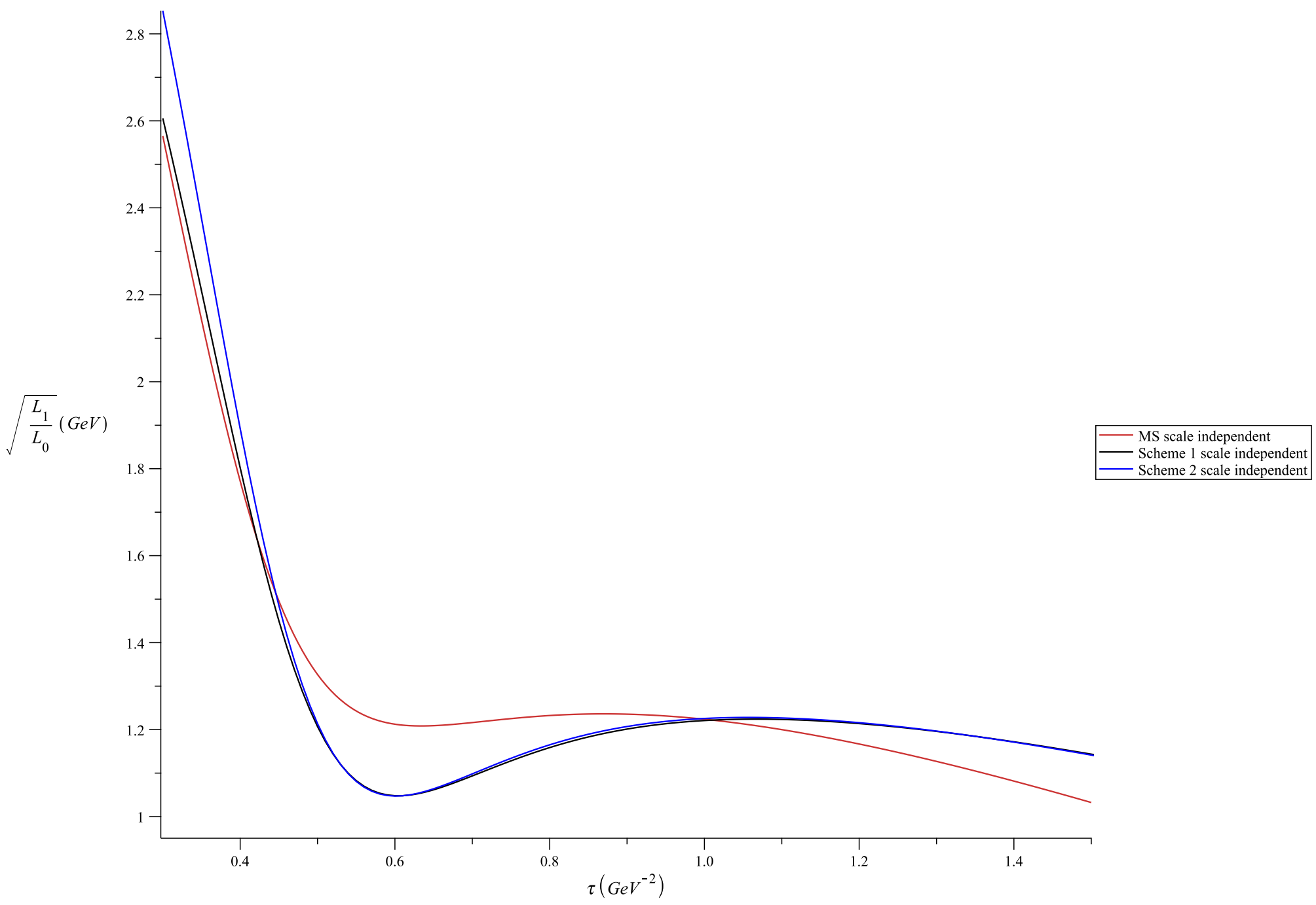

Figure 3: Scale independent scalar glueball mass bound as a function of $\tau$ in MS scheme, Scheme 1 and Scheme 2 